\title{
Le localisme Cynégétique à l'épreuve du Développement durable. Autochtonie et gestion des territoires dans la Somme
}

Sustainable development and hunting. Autochtonie and handling of the areas devoted to hunting in the Somme Valley

\section{Christophe Baticle}

\section{(2) OpenEdition \\ Journals}

Édition électronique

URL : http://journals.openedition.org/economierurale/3407

DOI : 10.4000/economierurale.3407

ISSN : 2105-2581

\section{Éditeur}

Société Française d'Économie Rurale (SFER)

Édition imprimée

Date de publication : 1 mars 2012

Pagination : 152-166

ISSN : 0013-0559

Référence électronique

Christophe Baticle, « Le localisme Cynégétique à l'épreuve du Développement durable. Autochtonie et gestion des territoires dans la Somme », Économie rurale [En ligne], 327-328 I janvier-mars 2012, mis en ligne le 01 mars 2014, consulté le 02 mai 2019. URL : http://journals.openedition.org/ economierurale/3407; DOI : 10.4000/economierurale.3407 


\section{Le localisme cynégétique à l'épreuve du développement durable Autochtonie et gestion des territoires dans la Somme}

Christophe BATICLE - Université de Picardie Jules Verne, Équipe Habiter - Processus identitaires, Processus sociaux, Amiens

"Entre la socialisation marchande de la nature et l'étatisation bureaucratique de ses usages ludiques, la voie vers une autogestion localisée de la chasse semble bien étroite... »

(Bages \& Nevers, 1982)

A u-delà de leurs rodomontades, visiblement antinomiques d'avec un mode d'appréhension écologique de la nature, les chasseurs (et parmi eux les plus viscéralement opposés aux « ayatollahs verts », à savoir les « sauvaginiers $»^{1}$ ) n'ont pourtant cessé de modifier leurs pratiques, jusqu'à récemment prétendre constituer un réseau de lanceurs d'alerte quant aux crises sanitaires affectant la faune dite sauvage.

En dehors donc des discours, nous nous proposons de montrer comment cette « écologisation » des actes se concrétise in situ, y compris au sein des bastions les moins disposés à concéder une quelconque valeur à l'analyse écologique du territoire local, dont ils font, par ailleurs, leur référent identitaire fondamental (Baticle, 2007).

À ce titre, le département de la Somme se révèle intéressant à bien des égards. Non seulement il apparaît comme une Mecque pour les adeptes du gibier d'eau, mais il se veut encore une terre de petit gibier, deux formes de pratique largement investies par les classes populaires et la paysannerie (Mischi, 2008). Longtemps on interpréta la première de ces modalités d'exercice à la manière d'une prédation sans vergogne pour une ressource considérée comme manne providentielle venue du ciel. De ce fait, son

1. À savoir ici les chasseurs de gibier d'eau pratiquant notamment dans des installations pérennes, dites « huttes », «gabions » ou « tonnes », situées en bordure de plans d'eau ou dans les baies. caractère gestionnaire fut jugé improbable (Pérès, 1998). Quant à la seconde, largement occupée par des «gens du pays », on l'assimila très vite comme à une catégorie regroupant des porteurs de fusil peu enclins à se préoccuper du renouvellement du cheptel et donc largement artificialisée (Fabiani, 1982).

C'est d'autant plus crédible que la régulation faunistique y est appréhendée comme un soutien à l'agriculture contre ses ravageurs et autres nuisibles. Sans compter que fief national du Mouvement de la ruralité (Bussi \& Ravenel, 2001, Bussi \& Fourquet, 2002 ; Le Bras, 2002 ; Baticle, 2010), la conception orgiaque d'une chasse cueillette trouva dans la Somme un débouché politique inattendu (Traïni, 2003).

Plus avant, c'est la question des postures apparemment antithétiques entre, d'une part stratégie de communication écologique (réactive et défensive) versus « verdissement » de l'ethos cynégétique qu'il s'agira de poser, en proposant une articulation moins binaire à cette alternative. On repère, en effet, deux grandes thèses cherchant à interpréter l'écologisation de la praxis en vigueur chez les « hommes en kaki » (Constanty, 2002). Du côté de Dalla Bernardina (1989), il ne s'agit ni plus ni moins que d'une invention discursive arrivée sur le tard, et ce dans le

2. Soit le parti CPNT : Chasse, Pêche, Nature et Traditions. 
Fidélité spatiale en années (hors non-réponses)

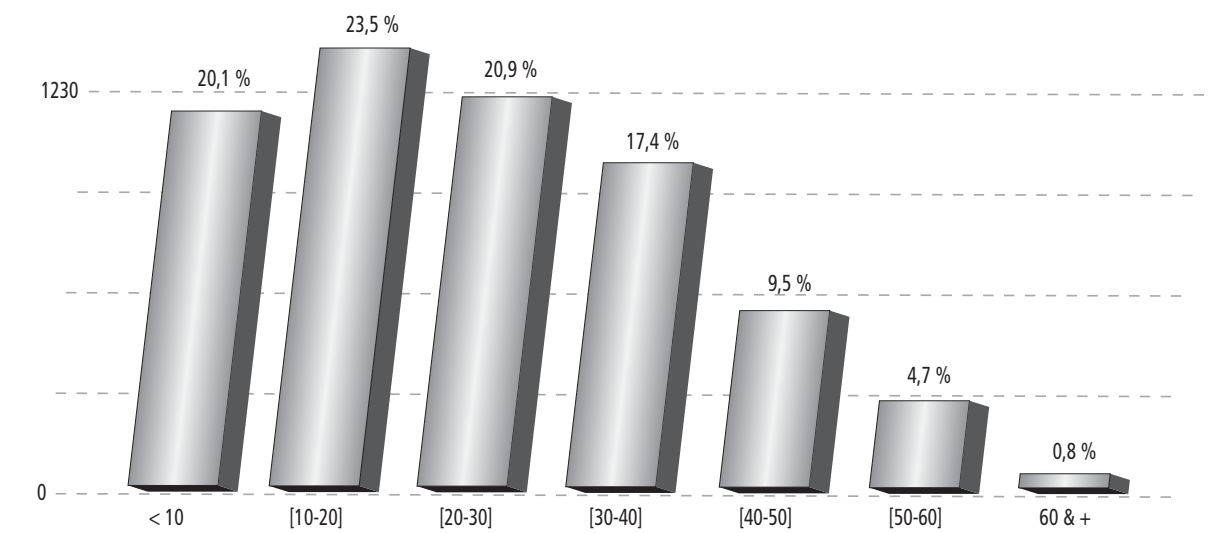

Min. $=1$ année - Max. $=80, X=22,97, \Sigma=14,67$

Source : Baticle, 2007

droit fil de l'analyse déconstructiviste des « traditions » (Hobsbawm \& Ranger, 1983 ; Blot, 2002 ; Babadzan, 2004). A contrario, chez Guimelli $(1989,1998)$ on déduit des observations monographiques une pratique écologique déjà ancienne et, par ailleurs, accompagnée d'une collaboration avec les associations environnementalistes au niveau local.

Si néanmoins les chasseurs affirment se compter parmi les premiers écologistes, force est de constater la distance entre leur conception d'une biodiversité très anthropocentrée et celle de leurs détracteurs. Aussi, la position qu'ils adoptent pour la protection des milieux favorables à la faune relève du recyclage des convictions anciennes plus à même de répondre aux injonctions nouvelles. Il ne s'agit donc ni d'un retournement opportuniste, ni d'un retour aux sources d'une chasse prétendument écologiste, mais bien d'une attitude visant à assurer les conditions d'une chasse durable. Aussi, même s'il existe une extrême variabilité des processus d'écologisation selon les groupes d'acteurs envisagés, on peut énoncer qu'une étroite articulation entre socio diversité et biodiversité oriente très efficacement les représentations dominantes quant aux usages légitimes de la nature qui dominent certains territoires (Manceron, 2005). En ce sens, certaines manières de penser l'acte de chasse (Bernard et al., 1998 ; Alphandéry et Fortier, 2007) peuvent contribuer au développement durable, même si c'est in fine la durabilité de la pratique elle-même qui se trouve visée.

Dans le cadre de cet article, nous chercherons d'abord à montrer comment les chasseurs de la Somme reflètent à l'égard du local une dimension autochtone qui ne se dément pas, pour ensuite illustrer leur implication à l'égard des territoires d'exercice, laquelle révèle les transformations en cours dans les manières de penser l'environnement. Enfin, il s'agira de réfléchir à ce que la socio diversité apporte en matière de biodiversité. Pour ce faire, nous prendrons appui sur les expériences comparatistes menées sur les sites Natura 2000 de la côte picarde, haut-lieu de la chasse au gibier d'eau (Baticle, 2003, 2004), en les replaçant dans le cadre plus global des données issues de la sociologie des chasseurs de la Somme (Baticle, 2006), soit un corpus de 5240 répondants, correspondant en 2006 à un cinquième des pratiquants du département. 


\section{La relation aux territoires Proximité et distance, propriété et milieux}

La chasse reste un puissant vecteur de socialisation dans les mondes ruraux et confère un « capital d'autochtonie » (Renahy, 2005) efficient dans les processus de légitimation des groupes en présence. La plupart du temps, ce processus passe par un territoire de prédilection. Une manière habituelle de mesurer l'incrustation des pratiquants consiste à s'interroger sur leur «fidélité » (graphique 1.) Une longévité dans l'espace qui ici ne doit pas s'entendre nécessairement comme une option retenue par préférence, même si c'est de la sorte que les édiles cynégétiques aimeraient pouvoir la comprendre. Etre attaché à un territoire peut en effet correspondre à une situation de réclusion; soit que l'on ne réussisse pas à s'intégrer à d'autres équipes de chasse (Weber, 1982), soit en raison de conditions financières inaccessibles (Pinet, 1990).

Maintenant, la connaissance empirique des chasseurs de la Somme nous permet d'affirmer qu'un lien étroit relie le processus de construction identitaire, dans et via l'exercice cynégétique, avec la pratique d'un territoire, résultat auquel on aboutit par ailleurs si on aborde la problématique sous un angle anthropo-cognitif (Warnier, 2009).

Dans l'ensemble, cette inscription spatiale maximale adopte un profil très parallèle à la répartition par classes d'âges. On peut de ce fait considérer la mobilité relativement faible pour au moins ce territoire de référence, souvent le premier dans la carrière du chasseur, ce qui nous permet d'appréhender la corrélation avec la longévité de la pratique, dont la moyenne s'élève à 27,5 années.

\section{L'autochtonie en question : chasser et se situer}

Parmi les chasseurs qui exercent dans la Somme, près des deux tiers sont domiciliés sur un territoire où ils pratiquent. Cette proportion s'avère importante, mais elle doit utilement être complétée par le quart des répondants chassant dans une commune limitrophe. Hors doubles comptes, ce sont ainsi 3826 répondants qui se placent dans une proximité évidente, soit les trois quarts. Toujours sans doubles comptes, 514 autres s'inscrivent au moins dans le canton, soit au total près de $83 \%$ de locaux.

En d'autres termes, il paraît logique, concernant une pratique comme la chasse, de s'interroger sur l'autochtonie dont témoignent les répondants. Celle-ci doit d'ailleurs moins s'entendre comme le fait de résider dans la commune de naissance au sens strict, que dans celle où l'on a vécu dès son plus jeune âge. Si cette hypothèse se confirmait, elle contredirait l'idée communément admise d'une désaffiliation territoriale au profit de la marchandisation des territoires cynégétiques (tableau 1).

Lorsque l'on connaît le turn over résidentiel de plus en plus élevé que mettent en visibilité les derniers recensements, l'on ne peut qu'être impressionné par le taux d'autochtonie déclaré, même en tenant compte de la précaution d'emploi ci-dessus mentionnée. Ainsi, plus des sept dixièmes déambuleraient entre autres territoires sur celui qui a marqué leur prime enfance. Ils

Tableau 1. À la question : « Parmi les territoires de chasse où vous pratiquez, l'un d'eux est situé dans une commune "

\begin{tabular}{l|c|c}
\multicolumn{1}{c|}{ Autochtonie } & Nbre cit. & Fréq. \\
\hline Non-réponse & 927 & $17,7 \%$ \\
\hline Où vous êtes né & 2261 & $43,1 \%$ \\
\hline Où vous avez passé une partie de votre enfance & 1433 & $27,3 \%$ \\
\hline Proche de votre commune d'enfance & 1761 & $38,6 \%$ \\
\hline Total obs. & $\mathbf{5 2 4 0}$ & \\
\hline
\end{tabular}

Source : Baticle, 2007. Calculs de l'auteur 
Tableau 2. Croisement entre autochtonie et communes de chasse

\begin{tabular}{l|c|c|c|c|c}
\hline Communes de chasse $\quad$ Autochtonie & $\begin{array}{c}\text { Non- } \\
\text { Réponse }\end{array}$ & Naissance & Enfance & $\begin{array}{c}\text { Proximité } \\
\text { Enfance }\end{array}$ & TOTAL \\
\hline Non- réponse & $7,8 \%$ & $1,7 \%$ & $3,7 \%$ & $2,3 \%$ & $3,4 \%$ \\
\hline Dans la commune où vous habitez & $42,8 \%$ & $78,5 \%$ & $64,7 \%$ & $54,9 \%$ & $62,1 \%$ \\
\hline Dans une commune limitrophe & $18,0 \%$ & $26,3 \%$ & $28,0 \%$ & $32,9 \%$ & $24,2 \%$ \\
\hline Dans une autre commune du canton & $22,2 \%$ & $23,8 \%$ & $25,9 \%$ & $31,8 \%$ & $24,9 \%$ \\
\hline Dans une autre commune de la Somme & $51,1 \%$ & $40,0 \%$ & $45,8 \%$ & $47,9 \%$ & $44,6 \%$ \\
\hline TOTAL & $100 \%$ & $100 \%$ & $100 \%$ & $100 \%$ & $100 \%$ \\
\hline
\end{tabular}

Dépendance très significative $-\mathrm{Chi}^{2}=417,97$, Degrés de liberté $=9,1-\mathrm{p}=>99,99 \%$.

Source : Baticle, 2007. Calculs de l'auteur. Les données encadrées révèlent des écarts significatifs

seraient encore près de $30 \%$ à disposer d'un droit de chasse dans une commune avec laquelle ils conservent un ancrage via la pratique cynégétique. Quant aux autres, la plupart ne se sont guère éloignés en pratiquant au moins dans un espace proche. Seul moins d'un cinquième ne se retrouverait pas dans ces modalités, toujours hors double-comptes ici.

Si l'on s'intéresse en premier lieu à ces non-réponses, on constate d'abord qu'il s'agit moins souvent de résidents du département, habitant des communes nettement plus peuplées, voire urbaines, alors qu'ils sont également citadins de par leur socialisation primaire. En conséquence, c'est leur accessibilité à un territoire qui se trouve mise à l'épreuve. Ainsi, un débat récurrent dans la sociologie de la chasse (Bozon, 1982) n'en a pas finit de persister, même après l'émergence d'une régulation marchande des loisirs. Il oblige à toujours réinterroger cette relation privilégiée entre site-s de la pratique et ressources socioterritoriales mobilisées afin d'y accéder (tableau 2).

Autre élément d'appréciation de cette autochtonie, le fait de pratiquer dans la commune de naissance s'associe fortement avec la chasse à domicile. C'est également dans ce croisement que l'on trouve l'effectif le plus élevé : 1776 citations, soit un tiers du total. S'en déduit, pour revenir aux non répondants évoqués précédemment, que ces derniers se distinguent en termes de statut cynégétique. Pour exemples, ils recourent plus souvent à l'actionnariat et sont moins régulièrement sociétaires de droit. De la même manière, ils entrent peu dans la catégorie des détenteurs d'un droit de chasse. Même lorsque l'accès est réglementé par un système d'adjudication, leur degré d'information les pénalise pour y concourir. Localement moins bien intégrés donc, ils occupent dans la chasse peu de rôles : garde-chasse, piégeur etc. Ce retrait s'exprime également sur les fonctions électives de direction des associations cynégétiques. Cette réalité différenciée a des conséquences sur le système de valeurs développé. Ainsi, la chasse représente d'autant plus un ancrage local que la pratique s'exerce sur un territoire d'enfance. Côté sociologique, les autochtones sont aussi plutôt moins diplômés, exerçant davantage les métiers d'agriculteur ou d'ouvrier. Ils sont enfin beaucoup plus souvent issus du milieu agricole.

Ces portraits hétérogènes peuvent être complétés entre autres par un questionnement relatif aux collègues de la pratique, la chasse prenant pour certains la forme d'un retour saisonnier au pays (Baticle, 2009), où l'on retrouve amis d'enfance et parents. Encore une fois, les extérieurs se distinguent très fortement des catégories de locaux, vérifiant l'hypothèse d'une intégration familiale au territoire chez ces derniers. On pratique ainsi d'autant plus avec des parents ou des connaissances de longue date que son territoire de chasse s'inscrit dans un espace plus proche du 
Figure 1. Répartition des professions et catégories socioprofessionnelles par \% décroissant des propriétaires

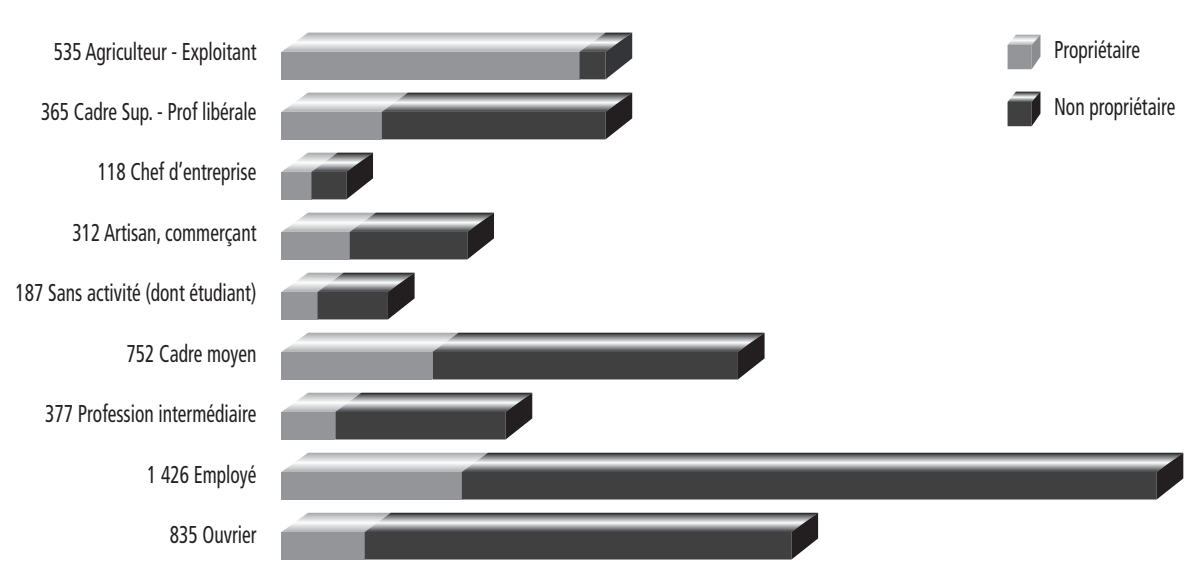

Dépendance très significative. $\mathrm{Chi}^{2}=1048,45$, Degrés de liberté $=8,1-\mathrm{p}=>99,99 \%$.

Source : Baticle, 2007

lieu de prime enfance. En revanche, les horsains cynégétiques exercent plus souvent seuls et culminent dans le statut de gendres accompagnant leurs beaux-parents.

En substance, les milieux cynégétiques se révèlent ici largement tributaires d'espaces signifiants dans le parcours biographique des intéressés, induisant chez ces derniers un processus de légitimation du rapport à la nature qui emprunte à la notion d'autochtonie un sens extensif et stratégique. Cette perception-appropriation de l'environnement proche offre un cadre idéal à l'identification territoriale, laquelle peut entrer en contradiction avec une définition plus globale de patrimoine universel.

\section{Les inégalités foncières : superficie et surface sociale}

Rappelons une lapalissade : la chasse exige avant toute chose de disposer d'un territoire. Or, ils sont jusqu'à un tiers à exercer sur certaines de leurs possessions foncières. On ne sera pas surpris d'apprendre qu'ils sont ici moins nombreux à éprouver des difficultés à trouver une assise, ces détentions, mêmes réduites, leur facilitant l'intégration dans un groupe de pratiquants (figure 1).

Surtout, les inégalités socioprofessionnelles devant la détention cadastrale sont suffisamment flagrantes pour y retrouver la pyramide sociale, hors secteur agricole bien entendu, dont le capital foncier représente l'outil de travail. Des inégalités qui se reproduisent parfaitement lorsque l'on considère la profession des pères. Pour autant, chez les locaux sans terre, on peut compenser ce handicap au travers du tissu relationnel, le capital social suppléant à la résidence au travers des invitations dans les communes environnantes.

Dimension complémentaire, le nombre de territoires communaux fréquentés révèle d'ailleurs la capacité des notables locaux à tisser un réseau d'ayants droit qui leur permettent de multiplier les espaces fréquentés (jusqu'à dix et plus dans les classes supérieures) ${ }^{3}$. Cette démultiplication a encore des conséquences financières qu'il convient de pouvoir soutenir, la moyenne du budget annuel croissant avec le nombre de communes fréquentées ${ }^{4}$. L'aisance spatiale combine de ce fait les deux déterminants que sont l'appartenance socioé-

3. L'écart-type est ici particulièrement significatif, avec 17,53 territoires pour une moyenne de seulement 2,47 .

4. À moins de $300 €$ annuels on se limite en moyenne à 1,48 territoire. Entre 1000 et $1500 €$ on passe à près de 5 . 
conomique et les opportunités familiales ou simplement résidentielles. On constate effectivement des classes populaires très présentes dans la modalité un seul territoire (près de $50 \%$ ), à l'inverse des chefs d'entreprises, des cadres supérieurs et des professions libérales (autour de $25 \%$ ), mieux représentés dans les tranches élevées. De la même manière, au fur et à mesure que croît la part du budget chasse consacré spécifiquement à l'accès aux territoires, ce sont également les surfaces qui augmentent, particulièrement en plaine et sur les zones boisées.

Une hypothèse concernant les évolutions à venir porte donc à imaginer un marché cynégétique dans lequel le territoire tendrait à devenir une variable régulée par l'étalon monétaire (Actéon, 1998). Il importe donc de connaître la façon dont ce chasseur devenu itinérant se comporte au regard de la distance parcourue pour atteindre ses différents terrains de pratique. Pour la Somme, l'ancrage sur au moins un territoire très proche (moins de $2 \mathrm{~km}$ ) reste la norme, les trajets les plus importants ( $50 \mathrm{~km}$ et plus) ne rassemblant que moins de $5 \%$ de l'effectif, soit généralement des citadins qui se rendent sur le domaine public maritime (DPM).

En résumé, l'autochtonie, ou tout au moins les relations établies de longue date avec le territoire, semblent plus que jamais caractériser cette population de chasseurs, mais le tourisme cynégétique des sauvagi- niers adhérents des associations du DPM permet de discerner une autre dimension plus visible : les porteurs de fusil ont commencé à entrer dans la société à habitants mobiles (Urry, 2005), celle d'une modernité où le réseau tend à supplanter l'inscription dans le local. Il y a ici un enjeu majeur en ce qui concerne l'économie de la chasse au sens des coûts d'accès aux territoires, mais également pour les modes de régulation en concurrence : entregent localiste versus financiarisation marchande. Nous verrons ci-dessous que les implications à l'égard de ces territoires sont hétéroclites, mais qu'elles prouvent une transformation de l'éthos cynégétique en direction d'une prise en considération des conditions de renouvellement de la faune. Toutefois, un critère proxémique continue de conditionner la disponibilité nécessaire à l'entretien des territoires.

\section{L'implication cynégétique L'accessibilité aux espaces}

\section{Les statuts du chasseur}

En vis-à-vis direct de cet enjeu central que constitue l'accessibilité aux territoires, le mode d'accès privilégié peut se déceler au travers du statut du chasseur (figure 2).

Près de la moitié de l'échantillon adhère au moins à une association de chasse dans la commune de résidence, répondant généralement à la définition d'une société

\section{Figure 2. Statut de l'accédant au territoire}

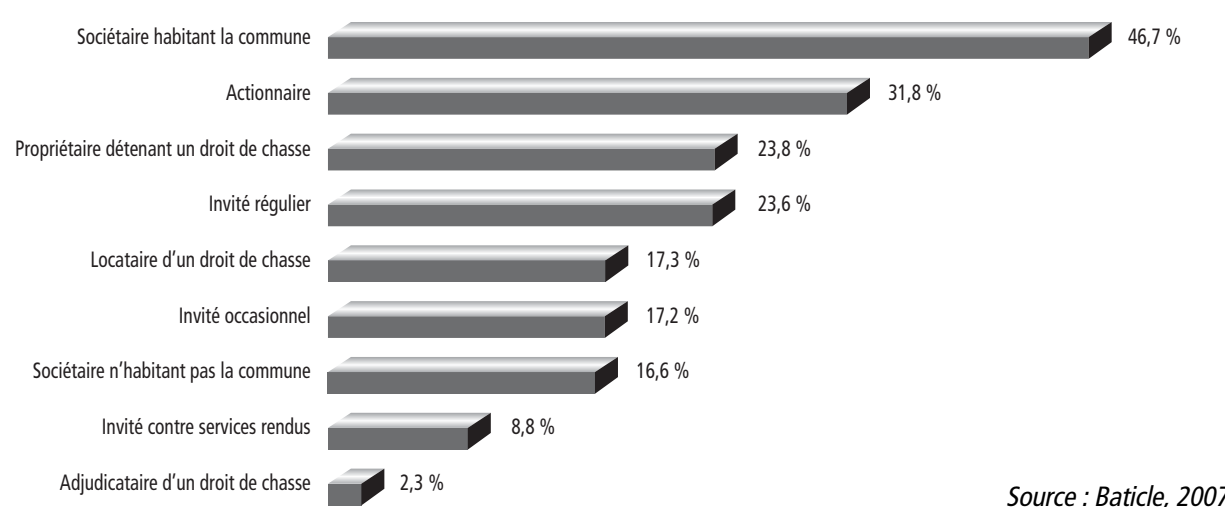


attribuant une facilité d'accès aux habitants, s'opposant par là à l'actionnariat. Actionnariat qui correspond justement à la deuxième situation rencontrée, soit par près du tiers de l'effectif. Face à cette alternative, deux moyens d'y échapper se présentent avec, d'une part la propriété (un quart) et, d'autre part, l'invitation à titre plus ou moins gracieux. Plus limitée, la location du droit de chasse revient avec l'adjudication à trouver un intermédiaire avantageux entre l'actionnariat simple et la propriété du sol.

Néanmoins, la multiplicité des situations provoque d'inévitables doubles comptes. De plus, un individu peut se trouver, selon le territoire considéré, dans différents cas de figure statutaires. Les 9918 citations amènent en effet à une moyenne de 1,9 statut par chasseur. Aussi, il convient de distinguer les situations en fonction des grandes catégories discernables :

- droit de chasse lié à la résidence : 2447 citations (46,7 \% des observations);

- droit de propriété impliquant de facto le droit de chasse : 1248 (23,8\%);

- droit de chasse tributaire d'une logique peu ou prou marchande : $2690(51,3 \%)$;

- logique de don et contre don : 2597 $(49,6 \%)$;

- autre logique : 871 (16,6\%).

\section{1. Être de quelque part et échapper au marché}

L'accès payant au territoire dispute de plus en plus fortement la première place à un modèle qui voulait voir dans le chasseur un habitant exerçant une captation de la faune sauvage locale sur la base d'un droit relevant plus de la communauté que du système sociétal moderne. Si l'on raisonne sur les citations, trois logiques dominent la population qui nous intéresse ici : marchande, résidentielle et basée sur les invitations (souvent croisées). En second lieu, près du quart de l'effectif dispose d'un droit de chasse lié à la propriété. Enfin, les sociétaires ne résidant pas dans la commune se situent dans des contextes complexes, dans lesquels la naissance, les liens familiaux et sociaux permettent d'échapper à l'actionnariat simple.

Néanmoins, certains statuts peuvent se suffire à eux-mêmes facilement, comme le fait d'adhérer à la société communale de son village sans chercher d'autre terrain de chasse $(20 \%)$. Les sociétaires n'habitant pas la commune $(4,5 \%)$, pour être généralement introduits au travers d'une forme plus ou moins formalisée de parrainage, épousent la plupart du temps les normes éthiques qui dominent localement. À l'opposé, les propriétaires ou locataires d'un droit de chasse peuvent se sentir plus libres à l'égard de la pression collective, mais ne pèsent que pour quelque $7 \%$ de l'échantillon. L'immense majorité doit tenir compte de la réalité d'un groupe qui peu ou prou impose ses modus operandi, quand ce ne sont pas ses modus vivendi. De façon similaire, le domicile, la propriété foncière et l'interconnaissance conditionnent donc très largement l'identité cynégétique par les statuts qui permettent d'accéder à un site disponible. Le seul jeu marchand explique rarement l'accès à un territoire, avec pour exemple un actionnaire sur cinq se résumant par cet unique statut (moins de 7\% du total).

D'une certaine façon c'est un nouveau pan du « marché cynégétique » qui s'effondre devant la nécessité d'un entregent plus facilement intronisant. Par ailleurs, la rencontre de l'offre et de la demande de chasse laisse en friche une équation plus complexe, voire contradictoire. D'un côté une demande plutôt issue des classes populaires, ici pléthorique, là insuffisante; de l'autre une offre qui peine parfois à répondre par un cheptel suffisant, alors qu'ailleurs la problématique achoppe sur une prolifération de sangliers devant un public insuffisant en nombre et vieillissant. C'est ainsi le binôme propriété-résidence qui assure à la chasse son noyau dur, quand la mobilité du chasseur devient l'exigence de la marchandisation des territoires. 
Tableau 3. Propositions d'actions pour l'entretien du territoire

\begin{tabular}{l|c|c}
\hline \multicolumn{1}{c|}{ Actions d'entretien } & Nbre cit. & Fréq. \\
\hline Non-réponse & 335 & $6,4 \%$ \\
\hline Agrainage & 3756 & $71,7 \%$ \\
\hline Comptage de gibiers & 3258 & $62,2 \%$ \\
\hline Implantation de cultures à gibiers & 1045 & $19,9 \%$ \\
\hline Plantations d'arbres et d'arbustes & 1468 & $28,0 \%$ \\
\hline Entretien d'allées forestières & 1567 & $29,9 \%$ \\
\hline Platières à bécassines & 570 & $10,9 \%$ \\
\hline Fauchage mare et territoire de hutte & 1467 & $28,0 \%$ \\
\hline Construction-aménagement de hutte ou des dépendances & 1242 & $23,7 \%$ \\
\hline Contruction-aménagement de « loge » ou des dépendances & 488 & $9,3 \%$ \\
\hline TOTAL OBS. & $\mathbf{5 2 4 0}$ & \\
\hline & Source : Baticle, 2007. Calculs de l'auteur
\end{tabular}

Le statut du chasseur répond encore à un fonctionnement propre aux milieux (nouveau test statistique très significatif). Pour exemple, la plaine étant principalement maîtrisée par des sociétés communales, on ne sera pas surpris d'y retrouver une surreprésentation des sociétaires résidents, alors que la situation inverse caractérise plutôt les zones boisées, sur lesquelles dominent l'actionnariat, l'appropriation privative et les variantes de l'invitation. Des propriétaires qui ont, semble-t-il, davantage tendance à se réserver le droit de chasse quand il s'agit de bois, contrairement à la plaine. Une réservation aux confins du finage gênant beaucoup moins la société communale, elle est plus facilement «tenable » face à la contrainte que font peser les chasseurs locaux sur les réservataires.

\section{Les rôles du chasseur}

Selon encore une fois leur statut, les chasseurs adoptent encore un comportement différent à l'égard du nombre de territoires communaux fréquentés. Aux extrémités, les communaux cantonnés à leur village face aux invités réguliers, passant d'une chasse à l'autre au gré des rencontres et des affinités nouées. Les propriétaires sont également souvent sédentaires par rapport à leur territoire, mais il leur faut généralement répondre à une invitation, quand eux-mêmes auront à garnir leur ligne de traque par leurs hôtes.

\section{Faire c'est être : travailler l'espace pour le marquer cynégétiquement}

Le principal impact écologique de la pratique cynégétique est peut-être moins à rechercher dans les «prélèvements », même si c'est cet euphémisme qui retient davantage l'attention, que dans les procédés mis en œuvre pour assurer l'attractivité des espaces. En ce sens, dans le débat sur le Développement durable (DD), c'est surtout l'interrogation de la chasse sur sa propre pérennité qui est posée (tableau 3).

Au total, 15196 citations permettent d'estimer que chaque répondant participe en moyenne à trois des neuf propositions d'actions portant sur l'entretien des territoires. En première ligne, l'agrainage et les opérations de comptage révèlent, si besoin était, le développement d'une chasse de plus en plus « cultivée », s'éloignant des formes dites «banales » qui dominaient dans les schémas classiques. Typiques de démarches plus récentes dans le temps, les plantations arborées et l'implantation des cultures à gibiers ont connu visiblement un certain succès.

Ces actions témoignent également d'un interventionnisme de plus en plus prégnant pour reconstituer les conditions de la « giboyeusité », pour reprendre le terme 
des techniciens en la matière. On repère encore les spécificités des modes de chasse comme celle du gibier d'eau ou du grand gibier, par rapport à l'association plaine et bois, souvent caractéristique des chasseurs en sociétés communales. Dans l'ordre, les adhérents de ces dernières regroupent près des deux tiers des citations, contre un peu plus d'un cinquième pour les sauvaginiers et moins de $15 \%$ pour les adeptes de la chasse forestière. Par exemple, un chasseur de marais sur deux a déjà procédé au faucardage, qu'il s'agisse de la végétation de la mare ou du territoire environnant. On en compte près de huit sur dix pour l'agrainage chez les communaux.

Toujours pour ce qui est de l'entretien des territoires, un tiers des répondants ne déclarent aucun travail bénévole à ce titre, ce qui en laisse environ 3500 pour réaliser près de 300000 heures annuelles. Si les variations sont fortes au sein des bénévoles, la moyenne représente néanmoins plus de deux semaines d'activité salariée. Même en retirant les valeurs qui paraissent relever davantage du professionnalisme, on reste à une moyenne de 75 heures par saison.

Quoi qu'il en soit, cette masse de travail reste élevée, ce qui semble confirmer des interventions régulières, non limitées aux périodes de chasse, contribuant à illustrer l'occupation régulière du territoire. On observe aussi et surtout deux pôles, dont l'un se cantonne à moins de $25 \mathrm{~h} / \mathrm{an}$, quand le second y consacre quatre fois plus de temps, voire davantage. Il y a ainsi visiblement plusieurs manières d'habiter (Lazzarotti, 2006) le territoire de chasse. Le critère qui semble le plus pertinent pour discriminer le temps de bénévolat relève bien du terrain. Les adeptes du gibier d'eau consacrent en moyenne 20 heures de plus que le chasseur lambda, alors que la plaine est le milieu qui paraît générer le moins d'engagement temporel.

Les plus engagés sont encore plus souvent des initiateurs et le nombre d'initiés croît avec la durée passée sur le terrain. Ils pratiquent plutôt en famille et comptent parmi les plus assidus. Ils résident plutôt sur place ou tout au moins à distance limitée de leur premier territoire de chasse. Il existe également une temporalité interne au temps du bénévolat, les plus jeunes pouvant être plus engagés que leurs collègues âgés de 20 à 40 ans, période la plus active de la vie professionnelle. L'approche de la retraite permet de repasser au-dessus de la moyenne et ce jusqu'à 70 ans, âge auquel les problèmes physiques font fortement baisser la durée du travail sur le terrain.

En parallèle à l'engagement temporel, l'investissement dans la chasse se concrétise encore par des rôles reconnus et parfois même institués. Au premier rang des fonctions découlant de la pratique, la garderie occupe 448 répondants, dont 108 cumulent terrain(s) privé(s) et surfaces relevant d'association(s) communale(s). Arrivés plus récemment, les piégeurs agréés correspondent au nouveau profil gestionnaire du chasseur.

Or, si aux trois quarts, les gardes sont aussi piégeurs, ces derniers sont souvent de simples chasseurs qui trouvent là une autre façon d'exister au sein d'un groupe. Près d'un répondant sur cinq déclarant s'adonner à cet exercice fastidieux et consommateur de temps, il convient de pousser plus loin les investigations sur les motivations exprimées par ce public spécifique, comptant selon les données fédérales 2007 plus de 4000 individus. Une série d'entretiens permet de comprendre ce qui pousse autant de pratiquants à appréhender le territoire de chasse dans la perspective d'un groupe qui le parcoure (Traïni, 2004). Si les fédérations ont mis en place une formation et un agrément spécifique à l'action du piégeur, force est de constater que ces dispositions ont rencontré sur le terrain un souci d'implication qui dépasse le discours sur la régulation. Encore en mai 2007, la revue fédérale de la Somme réalise une pleine page sur ce thème, avec un titre évocateur : «Piégeurs 
et chasseurs pour un même combat ", ce dernier mot étant imprimé en couleur. Le "piégeur digne de ce nom développe des trésors d'ingéniosité, de ruse et d'astuces qui confrontent son intelligence du genre humain à l'instinct de l'espèce animale ». Au sein des instances dirigeantes, on a ainsi fait des piégeurs et déterreurs "les "outils" de base, acteurs fondamentaux et préalables à toute politique de gestion ou tentative de maintien et développement des populations de gibier. On peut donc dire qu'il ne peut y avoir de gestion valable et durable sans piégeage ». Mais immédiatement à la suite de ces propos, le rédacteur en chef de la revue se sent l'obligation d'ajouter qu' «être piégeur ou déterreur est synonyme de dévouement » et surtout de relayer ce que ressasse depuis des années la commission fédérale chargée de travailler spécialement sur ce sujet, à savoir que «trop souvent encore on entend des piégeurs se plaindre du manque de reconnaissance de la part des chasseurs. Ceci explique, peut-être, que le département de la Somme qui compte 4588 piégeurs agréés, soit près de 6 par commune, n'en dénombre qu'à peine 1100 qui officient, soit un quart! ${ }^{6}$

Le paradoxe d'un engagement le plus souvent individuel, parfois en binôme mais toujours très prenant, nous ramène à la problématique développée par Garner (2003) à propos d'un autre public, par certains aspects en contradiction avec celuici, mais confronté au même dilemme sous l'angle adopté : comment trouver la satisfaction personnelle d'une action dont le résultat profite à tous. On pourrait même ajouter que contrairement aux « avocats de la cause animale », le paradoxe est double chez les chasseurs dans la mesure où ils se

\footnotetext{
5. Il est caractéristique ici que ce soit la commune qui face référence, et non l'effectif global des titulaires d'un permis de chasser validé. Le piégeur reste pensé comme "protecteur » d'un terroir. 6. Picardie chasse, $\mathrm{n}^{\circ} 114$, p. 12.
}

trouvent en concurrence les uns par rapport aux autres. Les enjeux du questionnement tiennent évidemment dans le statut du déterminisme amenant à l'engagement et portent à s'interroger sur les variables engagées dans cette détermination. Or, on ne sort des apories de cette apparente contradiction qu'en renonçant à la théorie des motivations proposée par Olson (1965). Ici, non seulement le piégeur n'est en rien un homo cynegeticus recherchant via ses actions la maximisation de son intérêt privé, mais qui plus est il n'a pas besoin de ces « incitations sélectives », sortes de rétributions indirectes à ses stratégies d'homme rationnellement porté vers le profit qui permettaient à l'économiste britannique d'expliquer l' « irrationalité » de la motivation pour le collectif. En ce sens et pour suivre la typologie de Garner, les sociétés de chasse sont davantage des groupes d'intérêts animés par une cause que des lobbies ordinaires. La cause, moins moraliste qu'anthropocentriste, qui anime le groupe des prédateurs humains, fait d'eux des entrepreneurs de ruralisme, par opposition aux entrepreneurs de morale (Becker, 1963) qui les affrontent sur le terrain des « droits imprescriptibles de l'animal ».

Il semble que le déterrage, même s'il est généralement et davantage défini comme une forme de chasse spécifique, peut être rapproché du piégeage d'un point de vue de l'inscription sociale d'un individu vis-à-vis d'un groupe. Dans les deux cas de figure, l'investissement physique est disproportionné par rapport aux prises et surtout ces dernières ne relèvent pas de la catégorie gibier. En d'autres termes, le piégeur et le déterreur se différencient sur le fait que l'exercice du second ne peut s'exprimer de façon solitaire, contrairement à celle du premier. Mais ils se ressemblent sur le plan de l'utilité collective qu'ils attribuent à leur quête. La recherche au sang, bien que beaucoup marginale, semble proche du déterrage en ce sens qu'elle constitue une spécialité, mais en prenant la question de la perte du gibier à l'autre extrémité. Quoi qu'il en 
soit, les équipages de chiens de rouge, comme on les appelle, entrent dans la même logique au profit d'une certaine éthique propre au monde cynégétique. Enfin, l'élevage bénévole, pratiqué par $7 \%$ de l'échantillon, peut s'entendre de la même manière, dans le sens d'un repeuplement du territoire qui pourra profiter au groupe. Les plus enclins à s'engager dans une forme d'action bénéfique au collectif ne sont pas non plus sociologiquement neutres. Les ouvriers sont par exemple les plus investis, surtout chez les gardes en société, à l'opposé donc des cadres supérieurs et professions libérales qui se font plus rares dans la majeure partie des engagements. Or, ces derniers ont pour autre caractéristique d'être surreprésentés parmi les chasseurs utilisant des chiens de rouge, laquelle apparaît ici comme une démarche distinctive, axée sur le pôle du « désintéressement ».

Des rôles qui ont bien entendu une incidence sur le temps consacré bénévolement à l'entretien des territoires puisqu'ils entrent dans les actions nécessaires au renouvellement des conditions de la pratique. On remarque à ce titre que gardes et piégeurs comptent parmi les mieux représentés dans les tranches temporelles les plus élevées.

\section{Biodiversité et socio-diversité}

La richesse des milieux entretenus par ces occupants territoriaux (en particulier sur les zones humides) ne doit néanmoins pas être confondue avec un quelconque altruisme à l'égard de la nature et c'est en quelque sorte sa force que de s'épauler sur leurs intérêts bien compris de Nemrod. On pourrait, pour la distinguer de l'engagement militant en faveur de la nature, parler de biodiversité sélective à visée cynégétique. C'est en effet la quête d'une catégorie de gibiers qui commande à l'entretien de ces espaces. Pour autant, l'aptitude à l'abnégation renvoie également à un intéressement pour le désintéressement. Selon les champs sociaux dans lesquels évoluent les agents, le don de soi peut entrer dans les logiques mêmes du moteur du champ. Aussi, parce qu'elle est fondamentalement une contestation radicale du tout économique, l'écologie politique ne pouvait se constituer sans laisser une large place à la nature pour la nature. Entre ces deux populations captivées par les mêmes milieux, dits « naturels », la différence se situe sur le plan de la vocation à leur réserver. Il s'agit donc bien ici d'une question éminemment politique quant aux choix qu'une société réalise en matière de dévolution des espaces. Si le chasseur contrarie sa pratique à venir en détruisant la ressource qui forme le fond de son exercice, la préservation des écosystèmes qui y sont associés ne peut pas être ignorée par lui. A contrario, nombre d'amoureux de la nature s'opposent à cette prédation humaine, soit par principe éthique au nom du «droit à la vie », décentrant ainsi l'organisation anthropocentriste du monde, ou encore en invoquant les risques qu'une sur-prédation ferait peser sur le maintien des cheptels. Autrement dit, l'élection d'une politique des territoires ne peut s'abstraire d'une réflexion quant aux représentations que se font les décideurs de la légitimité des discours s'opposant. Plus encore, au-delà des discours doivent être pris en compte les images dégagées par ceux qui les portent.

S'il fallait retenir une dimension caractérisant la modernité, la tendance à l'uniformisation tiendrait à n'en pas douter une place de choix. La globalisation des procédés de production, pour une fabrication de masse à destination d'un marché venant à s'internationaliser, s'est accompagnée d'une part de la spécialisation des producteurs et, d'autre part, du rapprochement entre les modes de vie. Stratification sociale et structuration de la production entretiennent ici des relations circulaires qui ont eu pour effet de mouler les espaces dans leurs logiques. À l'économie globale vient correspondre une spécialisation régionale, donnant aux territoires ruraux cette physionomie localement uniforme, qui porte 
l'empreinte de la tendance à la monoproduction. Or, la côte picarde, si elle est aujourd'hui interrogée pour les enjeux qu'elle soulève, a largement échappé à ce phénomène. La difficulté inhérente au drainage des marécages y est probablement pour beaucoup, bien qu'elle se soit davantage encore heurtée à la présence d'autres groupes d'intérêt et ici principalement à celui des sauvaginiers. S'en suit que toute réflexion sur la biodiversité implique de dégager au préalable les conditions de la socio-diversité qui en est à l'origine.

En la matière, la diversité sociale qui règne sur le littoral n'est plus à démontrer : pêcheurs à pied, mytiliculteurs, agriculteurs-éleveurs, bergers, chasseurs, touristes, résidents secondaires... ont produit ces paysages si singuliers par leur variété en un espace si restreint. Une diversité qui peut s'appréhender sous au moins deux angles : comme finalité ou comme moyen.

De façon plus empirique, l'étude des pratiques concrètes, comme celles qui consistent à aménager les territoires, montre que les transformations travaillant le verdissement de l'opinion (Bozonnet, 2005) sont également à l'œuvre chez les chasseurs, que ces derniers ne s'exemptent pas de cette réflexivité qui amène à se questionner sur les impacts de ses actes.

\section{Conclusion \\ La chasse dans le développement durable}

In fine, réfléchir la problématique cynégétique contemporaine dans la thématique du développement durable implique de faire le distinguo entre la biodiversité recherchée comme finalité de celle visée comme moyen. Dans des sociétés interpellées par le risque (Beck, 2001), le principe de précaution justifie à lui seul qu' elle soit appréhendée comme un objectif à maintenir. La question se pose assez différemment pour les chasseurs, selon que leurs espèces de prédilection sont menacées ou pas dans leur existence, sur les seuls terrains où ils pratiquent ou plus largement. En conséquence, davantage que le développement durable, c'est surtout la durabilité de la chasse qui est en jeu, sachant que la définition même de la pratique répond à des critères éminemment liés aux représentations légitimes que les pratiquants s'en font ici et maintenant. De cette distinction découle qu'un débat quant aux attendus des pratiquants serait nécessaire pour faire émerger cette définition normative de l'acte cynégétique légitime, et recevable pour le corps social dans sa globalité. Autant dire que ce sont les rapports de force entre chasseurs d'abord, puis entre ces derniers et la société englobante qui s'exprimeraient au travers de quelque conception que ce soit.

Il n'en va pas forcément différemment du développement durable. Tout d'abord, celui-ci n'a pas un sens unique, mais variable selon les objectifs que l'on vise à promouvoir. C'est donc une notion particulièrement stratégique, née de problèmes réels, mais traités selon les grilles de lecture des intérêts en jeu. La question sous-jacente reste celle de la répartition sociale des ressources dans un contexte de crise des modes de production basés sur la croissance.

Or, si dans nos pays la chasse n'est plus destinée à constituer un expédient alimentaire, on ne peut guère plus la considérer comme un loisir parmi d'autres, ni même exactement un loisir parce que se trouve posée dans son principe la question de la mise à mort, laquelle tend à constituer une ligne de fracture de plus en plus aigüe quant aux régimes de civilités. Il existe de multiples modalités apportées par la chasse au travers des âges pour y fournir des réponses socialement acceptables : les façons de tuer ont profondément changé, reflétant ces injonctions sociales. Les rituels magiques constituaient d'antan d'autres formes de justification, que le développement durable tend à remplacer désormais, 
comme lorsqu'une espèce prolifère à l'encontre des autres.

La prédilection pour une forme de chasse plutôt qu'une autre est très révélatrice de l'appartenance sociale des adeptes, mais également de leur parcours. Elle est encore liée à des contextes locaux. La plupart des subtiles distinctions qui font que la chasse ici, n'est jamais totalement la même que làbas, sur le plan des manières de faire, de se structurer, d'intégrer les membres, de poser des conditions éthiques; ces distinctions sont bien entendu le produit d'une histoire des rapports de force entre groupes présents sur le terrain, mais aussi une manière de faire valoir sa différence, de jouer des signes qui disent l'Ici (Jamin, 1979). Ce constat est assez logique dans la mesure où le moteur de la chasse n'est pas le gibier (ou pas seulement), mais davantage ce que des groupes d'acteurs sociaux localisés font du gibier (parfois d'un gibier plutôt qu'un autre), soit un intermédiaire pour régler un problème fondamental : comment donner une réponse symbolique à la manière d'être un homme. Ce qui revient à se demander comment établir la césure entre le sauvage et le civilisé. Mais également de poser la façon dont le «Nous, Ici » y apporte sa réponse propre, via un type de pratique, une manière de l'organiser, d'y intégrer les ayants-droit, de leur imposer des principes éthiques, des règles relatives au «bien tuer », etc.

Plus avant, Descola (2005) montre que cette manière de produire une spécificité humaine ontologiquement distincte des autres espèces vivantes, via et de par l'opposition entre nature et culture, n'est qu'une variante « civilisationnelle » locale, propre à l'Occident. Par là, on peut penser le développement durable comme un avatar parmi d'autres de ce même naturalisme qui amena à l'asservissement de la nature à l'aune des seuls intérêts humains. En d'autres termes, l'anthropologue héritier de Claude LéviStrauss revisite aussi le structuralisme de son maître en relativisant les universaux anhistoriques qui en faisaient le travers. Le schéma théorique qu'il échafaude part d'un principe cardinal : les cultures se définissent spécifiquement dans la façon dont elles envisagent la relation entre l'humain et le non-humain. À commencer par les attributs accordés à ce dernier : sur le plan physique d'une part, psychique de l'autre. L'animal par exemple, relève-t-il du même univers des atomes que le nôtre, tout en s'éloignant de la vie psychologique qui caractériserait l'Homme, sa réflexivité, ce que l'Occident judéo-chrétien tend à postuler ? Quoi qu'il en soit, pour Descola les quatre grands ordonnancements qu'il repère à travers le monde et l'histoire dans cette plus ou moins grande identité de nature que l'humain accorde au nonhumain, se fonde sur un invariant central : le dualisme du dedans et du dehors. Penser le différent implique de le placer en situation d'extériorité par rapport au soi, donc à se réfléchir comme un dedans, obligeant du même coup à se décentrer soi-même.

C'est la raison pour laquelle, à notre sens, la question du territoire est centrale. L'espace n'est pas seulement un réceptacle de la vie sociale, mais l'un des lieux où ses normes s'élaborent, se négocient et se débattent (Ostrowestky, 1995). Cadre dans lequel se construit la sociabilité, c'est-àdire où s'élaborent les manières du vivre ensemble, l'espace permet cette construction des codes sociaux, et puisque dans la chasse il est surtout question de territoire, rien d'étonnant à ce que ce territoire cynégétique soit un lieu de socialisation. Par ce rapport à l'espace, on apprend sa place dans la société et la place d'autrui, on se forge une identité dans et par ce cadre de projection des relations sociales, qui est encore un espace où ces dernières s'élaborent. En refusant de scinder représentations de la nature et actions sur celle-ci, Descola offre encore le moyen de réfléchir à l'articulation entre une théorie du territoire-reflet hypostasié de la société au sens de Durkheim et un pur intellectualisme du 
type de celui proposé par Lévi-Strauss (1962) pour interpréter le totémisme 7 . Pour répondre à la critique formulée par Héran (2007) quant au structuralisme de Descola,

7. La théorie de l'habitus chez Pierre Bourdieu ne recherche pas une articulation si différente entre l'histoire sociale incorporée et son expressionreconstruction dans la pratique des champs sociaux, ce que relève par ailleurs Héran (1987). peut-être faut-il accepter l'idée que les structures ont une histoire, ce qui n'enlève rien à leurs qualités descriptives.

Si l'on suit cette voie, on peut s'attendre à ce que les exigences du développement durable amènent à des appréhensions du territoire cynégétique qui, en retour, contribueront à transformer le regard des chasseurs sur les animaux. Notons dans ce sens que la catégorie «nuisibles » a déjà perdu une part de sa force de représentation.

\section{RÉFÉRENCES BIBLIOGRAPHIQUES}

Actéon (1998). Bilan final de l'étude de l'offre et de la demande cynégétique. Paris, La Documentation française, $34 \mathrm{p}$.

Alphandéry P., Fortier A. (2007). Gérer autrement la faune sauvage en France. L'élaboration des ORGFSH comme outils de conservation de la biodiversité. Sociologia Ruralis, $\mathrm{n}^{\circ} 47$, p. 42-62.

Babadzan A. (2004). L'invention des traditions et l'ethnologie : bilan critique. In Dimitrijevic D. (dir.) «Fabrication des traditions. Invention de modernité », Paris, MSH, p. 313-325.

Bages R., Nevers J.-Y. (1982). L'organisation locale de la chasse. Autodéfense collective et régulation des conflits. Études rurales, $\mathrm{n}^{\circ} 87-88$, p. 209-221.

Baticle C. (2010). Les violences cynégétiques : anthropocentrisme et déclassement social. In Schehr S., Klinger M. (dir.), « Lectures du conflit Strasbourg », Néothèque, p. 55-85.

Baticle C. (2009). La nature très sociale de l'environnement chez les chasseurs. Mode de vie et affirmations politiques du principe d'autochtonie. In Dobré M., Juan S. (dir.) «Consommer autrement. La réforme écologique des modes de vie », Paris, L'Harmattan, p. 199-216.

Baticle C. (2007). Les pratiques de chasse comme affirmations politiques du principe d'autochtonie. Dimensions territoriales des luttes cynégétiques. Thèse de socio- logie, Copans J., Kalaora B. (dir.), Amiens, UPJV, 989 p.

Baticle C. (2006). Les chasseurs de la Somme : portrait social. Amiens, UPJV, $190 \mathrm{p}$.

Baticle C. (2004). Chasse et environnement : implications réciproques? Amiens, UPJV, « Littoral et estuaires picards $», 520 \mathrm{p}$.

Baticle C. (2003). Chasse et environnement : implications réciproques? Amiens, UPJV « Marais Arrière littoraux picards », 690 p. Beck U. (2001). La société du risque. Sur la voie d'une autre modernité. Paris, Aubier, $521 \mathrm{p}$.

Becker H. (1985 [1963]). Outsiders. Études de sociologie de la déviance. Paris, Métailié, $248 \mathrm{p}$.

Bernard J.-L. (1998) (dir.). Les bords des champs cultivés : pour une approche cohérente des attentes cynégétiques, agronomiques et environnementales. Le Courrier de l'Environnement, $\mathrm{n}^{\circ} 34$, p. 21-32. Blot D. (2002). L'invention des traditionalismes. Étude anthropologique du développement des utilisations contemporaines de la notion de tradition. Contexte général et cas de la Picardie. Thèse de sociologie, Copans J. (dir.), Amiens, UPJV., 459 p.

Bozon M. (1982). Chasse, territoire, groupements de chasseurs. Études rurales, $\mathrm{n}^{\circ} 87$ 88, p. 335-342.

Bozonnet J.-P. (2005). Le verdissement de 
l'opinion publique. Sciences humaines, $n^{\circ} 49$, p. 50-53.

Bussi M., Fourquet J. (2002). La mosaïque politique de la France : 15 cartes par canton pour comprendre les élections présidentielles 2002. Cybergéo, http://www.cybergeo.fr.

Bussi M., Ravenel L. (2001). Écologistes des villes et écologistes des champs : analyse spatiale de l'implantation en France des partis écologistes et "Chasse, Pêche, Nature et Traditions ». Cybergéo.

Constanty H. (2002). Le lobby de la gâchette. Le pouvoir des chasseurs. Paris, Seuil, $212 \mathrm{p}$.

Dalla Bernardina S. (1989). L'invention du chasseur écologiste : un exemple italien. Terrain, $\mathrm{n}^{\circ} 13$, p. 130-139.

Descola P. (2005). Par delà nature et culture. Paris, Gallimard, 623 p.

Fabiani J.-L. (1982). Quand la chasse populaire devient un sport. La redéfinition sociale d'un loisir traditionnel. Études rurales, $\mathrm{n}^{\circ} 87-88$, p. 309-323.

Garner R. (2003). Le mouvement pour la protection des animaux aux États-Unis et en Grande-Bretagne. Politix, $\mathrm{n}^{\circ}$ 64, p. 75-102.

Guimelli C. (1998). Chasse et nature en Languedoc. Étude de la dynamique d'une représentation sociale chez les chasseurs languedociens, Paris, L'Harmattan, 207 p.

Guimelli C. (1989). Pratiques nouvelles et transformation sans rupture d'une représentation sociale : la représentation de la chasse et de la nature. In Beauvois J.-L. (dir.), «Perspectives cognitives et conduites sociales », Fribourg, Delval, vol. 2, p. 117-141.

Héran F. (2007). Vers une sociologie des relations avec la nature. $R F S$, vol. 48, p. 795-806.

Héran F. (1987). La seconde nature de l'habitus : tradition philosophique et sens commun dans le langage sociologique. RFS, vol. 28, p. 385-416.

Hobsbawm E., Ranger T. (2006 [1983]). L'invention de la tradition. Paris, éditions Amsterdam, $370 \mathrm{p}$.
Jamin J. (1979). La tenderie aux grives chez les ardennais du plateau. Paris, Musée de l'Homme, 158 p.

Lazzarotti O. (2006). Habiter, aperçu d'une science géographique. Cahiers de géographie du Québec, vol. 50, p. 85-102.

Le Bras H. (2002). Une autre France. Votes, réseaux de relations et classes sociales. Paris, Odile Jacob, 222 p.

Lévi-Strauss C. (1980 [1962]). Le totémisme aujourd'hui. Paris, PUF, 159 p.

Manceron V. (2005). Une terre en partage. Liens et rivalités dans une société rurale. Paris, MSH, 258 p.

Mischi J. (2008). Les militants ouvriers de la chasse. Éléments sur le rapport à la politique des classes populaires. Politix, $\mathrm{n}^{\circ} 83$, p. 105-131.

Olson M. (1978[1965]). Logique de l'action collective. Paris, PUF, $214 \mathrm{p}$.

Ostrowetsky S. (1995). Les quatre voies de l'identité. Identité, communauté, Paris, L'Harmattan, p. 23-33.

Pérès H. (1998). Entre désenchantement et réenchantement : chasser en Chalosse. Études rurales, $\mathrm{n}^{\circ}$ 147-148, p. 99-113.

Pinet J.-M. (1990). Les chasseurs à l'horizon 2000. Paris, INRA \& UNFDC, 190 p.

Renahy N. (2005). Les gars du coin. Enquête sur une jeunesse rurale. Paris, La Découverte, $284 \mathrm{p}$.

Traïni C. (2003). Les braconniers de la République. Les conflits autour des représentations de la Nature et la politique. Paris, PUF, 209 p.

Traïni C. (2004). Territoires de chasse. Ethnologie française, $\mathrm{n}^{\circ} 34, \mathrm{p} .41-48$.

Urry J. (2005 [2000]). Sociologie des mobilités. Une nouvelle frontière pour la sociologie ? Paris, Armand Colin, 253 p.

Warnier J.-P. (2009). Chasse et territoires : un apprentissage par corps. Colloque de la SFER, Clermont-Ferrand, ENITA, 13 p.

Weber F. (1982). Gens du pays, émigrés, étrangers : conflits autour d'une chasse en montagne. Études rurales, ${ }^{\circ}$ 87-88, p. 287-294. 